\section{AXILLARY ARTERY \\ CANNULATION PITFALLS}

\section{To the Editor:}

We read with interest the article by Orihashi and colleagues ${ }^{1}$ concerning axillary artery cannulation pitfalls. We present another possible drawback of axillary artery cannulation. A 69-yearold woman was admitted to the Department of Cardiac Surgery for a type A acute aortic dissection involving the innominate artery. After the usual cannulation of the right axillary artery, with a prosthetic side graft, and the right atrium, cardiopulmonary bypass (CPB) was established with no perfusion problems. At $26^{\circ} \mathrm{C}$ core temperature, $\mathrm{CPB}$ was arrested and the aorta was opened longitudinally. The entry tear was located in the ascending aorta. The innominate artery was clamped, and brain perfusion was begun through the axillary artery cannulation. The dissected aorta was excised, and a 30-mm prosthetic vascular graft was sutured to the distal aortic stump, just proximal to the innominate artery. The vascular graft was clamped, and, always via the axillary artery, systemic perfusion was restored. Near-infrared spectroscopy revealed poor cerebral perfusion, whereas the arterial line inflow pressure increased significantly. Suspecting an obstruction in the axillary artery or innominate artery, we directly cannulated the vascular graft and continued $\mathrm{CPB}$ antegradely. This allowed an uneventful completion of the procedure.

In aortic dissections involving the innominate artery, there may be a reentry

\footnotetext{
The Editor welcomes submissions for possible publication in the Letters to the Editor section that consist of commentary on an article published in the Journal or other relevant issues. Authors should: - Include no more than 500 words of text, three authors, and five references. - Type with double-spacing. - See http://jtcs.ctsnetjournals.org/misc/ifora.shtml for detailed submission instructions. - Submit the letter electronically via jtcvs.editorialmanager.com. Letters commenting on an article published in the JTCVS will be considered if they are received within 6 weeks of the time the article was published. Authors of the article being commented on will be given an opportunity of offer a timely response ( 2 weeks) to the letter. Authors of letters will be notified that the letter has been received. Unpublished letters cannot be returned.
}

tear in this vessel or the right carotid artery. Initially, this may not cause any problem. In fact, even if the axillary artery perfusion is partly directed through the reentry tear into the false lumen, this will empty through the proximal tear and malperfusion will not occur. But once the vascular graft has been sutured to the distal aortic stump and systemic perfusion is restarted, blood from the axillary artery cannulation may still enter the false lumen via the reentry tear in the innominate artery, but the proximal tear has been removed and there is a suture line instead. The false lumen is a blind pocket now, and it may dilate and obstruct the true lumen. To avoid this, after completion of the distal anastomosis of the prosthetic vascular graft, it is advisable to restore systemic perfusion antegradely, cannulating the vascular graft either directly or through a side branch. This is our current practice, and we strongly recommend it. In other words, during axillary artery perfusion serious complications may arise even from that short pathway that goes from the site of axillary artery cannulation to the origin of the innominate artery. We completely agree with Orihashi and colleagues ${ }^{1}$ that near-infrared spectroscopy represents an invaluable tool to monitor cerebral perfusion during surgical aortic procedures.

Giuseppe Rescigno, MD Carlo Aratari, MD, FECTS Marco L. S. Matteucci, MD Department of Cardiac Surgery Lancisi Hospital-Ospedali Riuniti di Ancona Ancona, Italy

\section{Reference}

1. Orihashi K, Sueda T, Okada K, Takahashi S. Compressed true lumen in the innominate artery: a pitfall of right axillary arterial perfusion in acute aortic dissection. J Thorac Cardiovasc Surg. 2009;137:242-3.

doi:10.1016/j.jtcvs.2009.02.045

\section{Reply to the Editor:}

We thank Rescigno and colleagues for their insightful comments regarding the pitfalls of axillary arterial perfusion. The case they presented illustrates another important mechanism of cerebral malperfusion under axillary perfusion. The surgical intervention can form a blind pocket (cul-de-sac) under retrograde perfusion, dilate the false lumen, and occlude the orifice of the right common carotid artery. We believe that the mechanism they postulated is right.

The "Y-junction" consisting of the innominate, right common carotid, and right subclavian (axillary) arteries is a tricky zone in aortic surgery, where cerebral malperfusion can be induced by various mechanisms: compression of the true lumen in the innominate artery in our case; blind pocked-induced malperfusion in the case of Rescigno and colleagues; and malposition of a selective perfusion cannula. ${ }^{1}$

An intimal flap is responsible for malperfusion not only in antegrade perfusion before surgery but also in retrograde perfusion during cardiopulmonary bypass. A switch between entry and reentry under retrograde perfusion creates a new perfusion status in this Y-junction. Surgical intervention can generate another change as Rescigno and colleagues mentioned. However, preoperative assessment is unlikely to be helpful for predicting an occurrence of malperfusion in the altered situation.

They proposed 2 solutions for this problem: direct cannulation of the aortic graft or the use of a branched graft to establish antegrade perfusion. The only drawback of these solutions is the necessity of an additional cannula or branched graft in every case for a rare complication. Extra safeguard is needed because intravascular events are not visible. In an attempt to elucidate the events in the blind zone, we established a method of visualizing arch branch arteries by means of transesophageal echocardiography. ${ }^{2}$ It was shown that morphologies and blood flow in the branch arteries, as well as the mechanism of malperfusion, vary considerably among individual patients. With such information, however, it is hardly possible to predict an occurrence of malperfusion under axillary artery perfusion. 
Our current strategy is to "perfuse and watch" with the aid of real-time monitoring and diagnostic imaging. As Rescigno and colleagues mentioned, near-infrared spectroscopy (NIRS) is useful for detecting newly developed cerebral malperfusion. Although they recognized an undesirable situation by inflow pressure and NIRS data, the former may not be apparent if the intima breaks at the suture line and pressure elevation is not apparent. Decreased oxygen saturation is likely to be a reliable indicator of an occurrence of something undesirable, whatever the cause.

Once malperfusion is detected, it is necessary to develop an appropriate strategy for restoring cerebral perfusion based on the cause of malperfusion. In this regard, visualization of the "Y-junction" with transesophageal echocardiography can be helpful for correctly pursuing the strategy, because it provides real-time information on morphology and perfusion.

However, the decision can be incorrect, as in our case. Thus, the decision needs to be immediately assessed to avoid further delay in restoring adequate perfusion. This can be achieved by NIRS. If oxygen saturation remains low, other possible scenarios should be considered.

We believe that a sequence of "detection, decision, assessment' is important in the operating room, especially in cases of acute aortic dissection. To minimize " unfortunate events of unknown cause," it is necessary to fully use the modalities available for obtaining realtime information. This will be another important task for cardiovascular surgeons, as well as surgical skill.

Kazumasa Orihashi, MD

Taijiro Sueda, $M D$

Kenji Okada, MD

Shinya Takahashi, MD

Hiroshima University Hospital

Hiroshima, Japan

\section{References}

1. Orihashi K, Sueda T, Okada K, Imai K. Malposition of selective cerebral perfusion catheter is not a rare event. Eur J Cardiothorac Surg. 2005;27:644-8.
2. Orihashi K, Matsuura Y, Sueda T, Watari M, Okada K, Sugawara Y, et al. Aortic arch branches are no longer a blind zone for transesophageal echocardiography: a new eye for aortic surgeons. J Thorac Cardiovasc Surg. 2000;120:466-72.

doi:10.1016/j.jtcvs.2009.03.028

\section{ENDOSCOPIC TREATMENT OF AIRWAY STENOSIS AFTER LUNG TRANSPLANTATION To the Editor:}

We read with interest the article from Thistlethwaite and colleagues ${ }^{1}$ entitled "Airway stenoses after lung transplantation: incidence, management and outcome.',

We agree that silicone stents are extremely useful to complete treatment of posttransplantation airway stenosis after mechanical/laser debridement; they can effectively support the airway during healing and prevent recurrence. The use of this type of stent is particularly indicated for type 1 and 2 stenosis (according to the classification reported by the authors), and they can be easily fenestrated to allow ventilation to the upper lobes when the bronchial stenosis comes across them. However, we have met some difficulties in placing these stents when the stenosis is extremely long and tortuous, encompassing also the airway distal to the anastomosis for a long segment or the peripheral airway (type 3 and 4 stenosis). This is obviously a very small group of patients (in the reported series there are only 3 [15\%] patients), but this problem has been certainly met by all the groups performing lung transplantation, and the difficulties in its treatment are evident. When this type of complication occurs, it usually requires several treatments with unsatisfactory results, and permanent stenting is usually indicated. In this situation we have found extremely useful the use of covered expandable metallic stents (Ultraflex; Boston Scientific, Galway, Ireland). They can be easily deployed, completing airway dilatation, and present a better ratio between the thickness of the wall of the stent and the diameter of the stent itself, allowing a better airflow, especially for small diameters; thus clearance of secretion is improved. Although they are more expensive than silicone stents and clearly more difficult to remove, their use might be preferred in this subset of patients. We have used them in 5 patients without major complications.

Could the authors provide more details about the group of patients with type 3 and 4 stenosis?

Marco Anile, $M D$
Tiziano De Giacomo, MD
Federico Venuta, MD
University of Rome 'Sapienza"
Policlinico Umberto I
Rome, Italy

Reference

1. Thistlewaite PA, Yung G, Kemp A, et al. Airway stenoses after lung transplantation: incidence, management, and outcome. J Thorac Cardiovasc Surg. 2009; $136: 1569-75$.

doi:10.1016/j.jtcvs.2009.01.012

\section{Reply to the Editor:}

We appreciate the interest that Drs Anile and Ventura have in the management of lung transplant recipients with distal focal bronchial stenosis (type 3 disease) and diffuse distal stenosis (type 4 disease). This is a complex group of patients to manage, and their airway issues can be challenging. ${ }^{1}$

As a high-volume center for bronchial stent placement (for causes other than transplantation), we have been uniformly disappointed with the use of expandable metallic stents. Metallic stents in the airway are expensive and associated with fracture, overgrowth of granulation tissue, and erosion through the tracheobronchial wall. Although the cost of a single metallic stent is low in comparison with the total expense of lung transplantation, cost precludes having a large inventory of these stents available. We currently have an inventory of more than $100 \mathrm{Si}$ lastic stents (Hood Laboratories, Pembroke, Mass, and Bryan Corporation, Woburn, Mass). This inventory allows us to find the right stent for almost any airway, to tailor a specific stent to an 